\title{
CLIMA DE SEGURANÇA E A PRÁTICA DE HIGIENE DAS MÃOS: PERCEPÇÃO DE TRABALHADORES E GESTORES
}

\section{SAFETY CLIMATE AND HAND HYGIENE PRACTICE: PERCEPTION OF WORKERS AND MANAGERS}

\section{PRÁCTICA DE SEGURIDAD CLIMÁTICA E HIGIENE DE MANOS: PERCEPCIÓN DE TRABAJADORES Y GESTORES}

\section{RESUMO}

Objetivo: avaliar o clima de segurança entre trabalhadores de saúde de ambientes críticos e a percepção de gestores sobre as práticas de higiene das mãos. Método: trata-se de um estudo transversal e analítico, realizado com profissionais de saúde $(n=142)$ e gestores $(n=54)$ de um hospital público. A equipe assistencial respondeu ao Safety Attitudes Questionnaire (SAQ) e os gestores ao questionário recomendado pela OMS sobre higiene das mãos. Resultados: observou-se que a percepção do clima de segurança esteve comprometida em todos os domínios avaliados, com pior avaliação para a Percepção da gerência. Para os gestores, há pouco comprometimento dos profissionais de saúde com a prática de higiene das mãos e, além disso, $37 \%$ dos gestores não acreditam que a participação do paciente seja ação eficaz na melhoria dessa prática. Conclusão: constatou-se que o clima de segurança deficitário e a percepção limitada sobre higiene das mãos são uma dualidade que pode comprometer a segurança do paciente.

Descritores: Segurança do Paciente; Higiene das Mãos; Equipe de Assistência ao Paciente; Organização e Administração; Controle de Infecções; Pessoal de Saúde.

\section{ABSTRACT}

Objective: to assess the safety climate among health workers in critical environments and the perception of managers about hand hygiene practices. Method: this is a cross-sectional and analytical study, carried out with health professionals $(n=142)$ and managers $(n=54)$ from a public hospital. The assistance team answered the Safety Attitudes Questionnaire (SAQ) and the managers answered the questionnaire recommended by WHO on hand hygiene. Results: it was observed that the perception of a security climate was compromised in all domains evaluated, with a worse evaluation for the Perception of management. For managers, there is little commitment by health pro- 
fessionals to the practice of hand hygiene and in addition, $37 \%$ of managers do not believe that patient participation is effective action in improving this practice. Conclusion: it was found that the deficient safety climate and the limited perception of hand hygiene is a duality that can compromise patient safety.

Descriptors: Patient safety; Hand Hygiene; Patient Assistance Team; Organization and Administration; Infection Control; Health Personnel.

\section{RESUMEN}

Objetivo: evaluar el clima de seguridad entre los trabajadores de la salud en entornos críticos y la percepción de los gestores sobre las prácticas de higiene de manos. Método: se trata de un estudio transversal y analítico, realizado con profesionales de la salud $(n=142)$ y gestores $(n=54)$ de un hospital público. El equipo de asistencia respondió el Cuestionario de Actitudes de Seguridad (SAQ) y los gestores respondieron el cuestionario recomendado por la OMS sobre higiene de manos.

Resultados: se observó que la percepción de clima de seguridad se vio comprometida en todos los dominios evaluados, con una peor evaluación para la Percepción de gestión. Para los gestores hay poco compromiso por parte de los profesionales de la salud con la práctica de la higiene de manos y además, el $37 \%$ de los gestores no cree que la participación del paciente sea una acción efectiva para mejorar esta práctica. Conclusión: se encontró que el clima de seguridad deficiente y la percepción limitada de la higiene de manos es una dualidad que puede comprometer la seguridad del paciente.

Descriptores: Seguridad del paciente; Higiene de manos; Equipo de asistencia al paciente; Organización y administración; Control de infección; Personal de Salud.

1Universidade Federal de Mato Grosso (UFMT). Cuiabá (MT), Brasil. 1® https: //orcid.org/0000-0002$8400-3684$

²Universidade Federal de Mato Grosso (UFMT). Cuiabá (MT), Brasil. 2『 http://orcid.org/0000-00027895-5435

${ }^{3}$ Universidade Federal de Mato Grosso (UFMT). Cuiabá (MT), Brasil. ${ }^{3}$ h https://orcid.org/0000-0002$2160-508 X$

${ }^{4}$ Universidade Federal do Rio Grande do Sul (UFRGS). Porto Alegre (RS), Brasil. 4[0 https: / / orcid.org/0000-0002-1822-2360

${ }^{5}$ Universidade Federal de Mato Grosso (UFMT). Cuiabá (MT), Brasil. ${ }^{5}$ ๑https://orcid.org/0000-0002- 
¿Universidade Federal de Mato Grosso (UFMT). Cuiabá (MT), Brasil. []ㅐ https://orcid.org/0000-00030126-3040

7Universidade Federal de Mato Grosso (UFMT). Cuiabá (MT), Brasil. ${ }^{7} \mathbb{B}$ http://orcid.org/0000-0002$2746-1865$

\section{Como citar esse artigo \\ Bezerra TB, Baggio E, Valim MD, Azevedo IVR, Oliveira JLC, Marcon SR, Bortolini, J. Clima de segurança e a prática de hygiene das mãos: percepção de tra- balhadores e gestores. Rev enferm UFPE on line. 2021;15:e247896 DOI: https://doi.org/10.5205/1981-8963.2021.247896}

\section{INTRODUÇÃO}

A segurança do paciente é um dos seis atributos da qualidade do cuidado e tem adquirido, em todo o mundo, grande importância na legitimidade da assistência qualificada1.

A fim de fortalecer o cenário da segurança na assistência, a Organização Mundial da Saúde (OMS) e a Joint Commission International $(\mathrm{JCl})$ criaram as metas internacionais de segurança do paciente para promover melhorias específicas em algumas áreas do cuidado e proporcionar uma assistência mais segura ${ }^{2}$. Dentre as referidas metas, encontra-se a necessidade de higienizar as mãos para prevenção das Infecções Relacionadas à Assistência à Saúde (IRAS).

As IRAS afetam milhões de pessoas em todo o mundo e representam um grande risco para a segurança do paciente devido aos altos índices de mortalidade e à elevação de custos às instituições e aos sistemas de saúde ${ }^{3}$.

Concorda-se, em todo o mundo, que a principal medida para prevenção das IRAS é a Higiene das Mãos $(\mathrm{HM})^{3-4}$. Dessa forma, a estratégia multimodal de melhoria da HM, divulgada pela Organização Mundial da Saúde em 2009, é composta por cinco componentes-chave: (1) Mudança no sistema: visa garantir a infraestrutura necessária para prática de HM; (2) Formação/educação: propõe treinamentos dinâmicos e periódicos a todos os profissionais da equipe; (3) Avaliação e feedback: monitoramento das práticas de HM e retorno dos dados aos profissionais; (4) Lembretes no local de trabalho: afixação de cartazes sobre a importância da HM; e (5) Clima de segurança institucional: criação de um ambiente favorável às questões de segurança do paciente, com participação ativa individual dos profissionais e feedback nos níveis individual e organizacional, além de apoio da gerência sênior e envolvimento de líderes institucionais ${ }^{2-4}$.

Atualmente, passados dez anos do lançamento da referida estratégia multimodal, sabe-se que os índices de adesão à higiene das mãos prevalecem deficientes em todo o mundo ${ }^{4}$ e estudos 
mostram pouco envolvimento da gestão na temática ${ }^{4-7}$.

Nesse contexto, ressalta-se que o apoio gerencial é crucial para implementação eficaz da estratégia multimodal de melhoria da HM, uma vez que esse componente é transversal a todos os demais elementos da referida estratégia e contribui substancialmente para a cultura de segurança do paciente. Estudos experimentais comprovaram que o envolvimento da gestão na referida estratégia multimodal de HM para construção de um clima favorável à segurança do paciente é primordial para obtenção de índices de adesão satisfatórios ${ }^{4}$.

A cultura de segurança é definida como o produto de valores, atitudes, percepções e competências, grupais e individuais, que determinam um padrão de comportamento e comprometimento de segurança da instituição, substituindo a culpa e a punição pela oportunidade de aprender com as falhas. O clima de segurança se refere aos componentes mensuráveis da cultura de segurança, refletindo a concepção dos profissionais em relação às questões de segurança do paciente na instituição1.

Diante disso, o Safety Attitudes Questionnaire (SAQ) se destaca como um instrumento válido capaz de proporcionar informações em relação aos elementos que necessitam ser implementados em instituições hospitalares para a segurança do paciente por meio da mensuração do clima de segurança entre profissionais da equipe de saúde e de domínios como Clima de trabalho em equipe, Clima de segurança, Satisfação no trabalho, Percepção do estresse, Percepção da gerência e Condições de trabalho ${ }^{8}$. Destaca-se que estudos têm evidenciado baixa percepção de clima de segurança do paciente, tanto em nível nacional ${ }^{6-8}$ quanto mundial ${ }^{9}$.

Acredita-se que investigar a percepção do clima de segurança em instituições de saúde com a visão de gestores sobre a importância da HM pode colaborar no planejamento e na efetividade de ações prioritárias para prevenção e controle das IRAS. Dessa forma, elaboraram-se as seguintes perguntas de pesquisa: qual a perceção do clima de segurança do paciente entre profissionais de saúde que atuam em ambientes críticos? Qual a percepção dos gestores sobre a efetividade das ações de higiene das mãos?

\section{OBJETIVO}

Avaliar o clima de segurança entre trabalhadores de saúde de ambientes críticos e a percepção de gestores sobre as práticas de higiene das mãos.

\section{MÉTODO}

Trata-se de um estudo quantitativo, do tipo transversal, analítico, realizado com todos os 
profissionais de saúde atuantes em unidades críticas de um hospital universitário do Centro-Oeste do Brasil. A instituição é de médio porte e presta serviços em nível terciário.

A população do estudo foi composta por profissionais de saúde e gestores. Os profissionais de saúde $(\mathrm{N}=180)$ representados por enfermeiros, técnicos de enfermagem, médicos, médicos residentes e fisioterapeutas atuantes nos setores de UTI Neonatal, UTI Adulto e Clínica Médica (ala de cuidados semi-intensivos), nos períodos matutino, vespertino e noturno, em todos os dias da semana. Os profissionais gestores $(\mathrm{N}=61)$ eram os colaboradores que ocupavam a posição de administrador, chefe de setor ou unidade.

Estabeleceram-se como critérios de exclusão trabalhadores que estavam afastados do setor de trabalho ou função gerencial, por motivo de férias ou licença médica durante o período da coleta de dados, e aqueles que possuíam menos de seis meses de experiência na instituição. Esse período justifica-se pelo tempo necessário para mensurar o clima de segurança organizacional, uma vez que a percepção dos trabalhadores diz respeito às questões de segurança em seu ambiente laboral ${ }^{8}$

Após concordância em participar da pesquisa mediante assinatura do Termo de Consentimento Livre e Esclarecido (TCLE), todos os participantes receberam os questionários e sua via do TCLE em envelopes opacos, ficando acordada uma data de retorno do pesquisador para buscá-los.

Os dados foram coletados nos meses de julho a setembro de 2018. Utilizou-se para tanto o Safety Attitudes Questionnaire (SAQ) Short Form 2006, adaptado a validado para realidade de hospitais brasileiros ${ }^{8}$. Para a coleta de dados com os gestores, optou-se por um instrumento validado pela OMS: Questionário sobre a percepção de Gerentes Executivos/Diretores/Administradores/Chefes sobre as infecções relacionadas à assistência à saúde e à higienização das mãos . Os dois questionários são autoaplicáveis, com tempo médio de preenchimento de 20 minutos.

O SAQ possui 41 itens que se propõem a mensurar o clima de segurança por meio de seis domínios: clima de trabalho em equipe; clima de segurança; satisfação no trabalho; percepção do estresse; percepção da gerência (do setor de trabalho e do hospital); e condições de trabalho. Trata-se de escala ordinal do tipo Likert, de 5 pontos: discorda totalmente a concorda totalmente. 0 escore varia de 0 a 100 pontos e são considerados valores positivos os escores $\geq 75$. O estudo de validação mostrou consistência interna satisfatória, com alfa de Cronbach de 0,89, e 
a análise fatorial discriminou os seis domínios supracitados .

O questionário da OMS é composto por 18 itens, sendo que as questões de 9 a 12 estão dispostas no formato de escala ordinal do tipo Likert de 4 pontos (1 - Muito baixo a 4 - Muito alto) e mensuram o impacto das IRAS na evolução clínica do paciente e nas despesas do hospital; eficácia da HM na prevenção de IRAS; e importância da HM nas prioridades da gerência. As questões 14 e 16 a 18 estão dispostas no formato de escala ordinal do tipo Likert de 5 pontos. A questão de número 14 (1 - Nem um pouco a 5 - Muito bons), a questão 16 (1 - Nenhum esforço a 5 - Grande esforço), a questão 17 (1 - Nem um pouco a 5 - Muito bem) e a número 18 (1 - Ineficaz a 5 - Muito eficaz) avaliam enfermeiros e médicos como bons exemplos para a promoção da HM na instituição; o esforço necessário dos trabalhadores de saúde para HM; a percepção do pedido para uma excelente HM pelos trabalhadores de saúde; e a eficácia das ações no aumento permanente da adesão às práticas de HM na instituição ${ }^{5}$.

Os dados foram tabulados no software Microsoft Office Excel $\circledast$ com dupla digitação independente. Após a confirmação de fidelidade, os dados foram analisados utilizando-se estatística descritiva para sumarizar o conjunto de dados obtidos. As variáveis categóricas nominais foram descritas e apresentadas em tabelas de frequência absoluta (n) e relativa (\%). As variáveis quantitativas foram expressas por média e desvio padrão. Testou-se a normalidade dos dados por meio do teste de Shapiro-Wilk e uma vez que se trataram de dados não normais realizou-se a comparação entre a percepção do clima de segurança e as categorias trabalhadores com o teste de Kruskal-Wallis no software R.

Este estudo faz parte de um projeto matricial intitulado "Estratégia Multimodal para a adesão dos trabalhadores de saúde à higienização das mãos: estudo quase-experimental”, aprovado pelo Comitê de Ética em Pesquisa da instituição sob parecer $\mathrm{n}^{\circ} 2.441 .333$ com número do Certificado de Apresentação para Apreciação Ética (CAAE) n 75169317.0.0000.5541, de forma que todas as prerrogativas éticas da resolução n $466 / 2012$ do Conselho Nacional de Saúde fossem cumpridas.

\section{RESULTADOS}

Do total de profissionais de saúde $(\mathrm{N}=180), 11(6,1 \%)$ não foram encontrados durante 0 período da coleta de dados, 10 (5,5\%) recusaram-se a participar do estudo, 8 (4,4\%) não devolveram o questionário após três tentativas, 7 trabalhadores $(3,8 \%)$ estavam de férias e 2 $(1,1 \%)$ de licença médica.

Do total gestores $(\mathrm{N}=61), 3(4,9 \%)$ estavam de férias, $3(4,9 \%)$ não foram encontrados 
exercendo a função mencionada e $1(1,6 \%)$ encontrava-se de licença-maternidade. Com isso, 196 profissionais compuseram a amostra final do estudo, representada por 142 trabalhadores de saúde assistenciais, sendo 60 técnicos de enfermagem, 24 enfermeiros, 29 médicos, 17 médicos residentes, 12 fisioterapeutas e 54 gestores, com taxa de resposta de $78,8 \%$ e $88,5 \%$, respectivamente.

Tabela 1 - Perfil dos participantes do estudo ( $n=142)$. Região Centro-Oeste, Brasil, 2018

\begin{tabular}{|c|c|c|}
\hline Variáveis & $f$ & $\%$ \\
\hline \multicolumn{3}{|l|}{ Sexo } \\
\hline Masculino & 45 & 31,70 \\
\hline Feminino & 97 & 68,30 \\
\hline \multicolumn{3}{|l|}{ Faixa Etária - (anos) } \\
\hline $20-30$ & 29 & 20,42 \\
\hline $31-40$ & 68 & 47,89 \\
\hline $41-50$ & 35 & 24,65 \\
\hline $51-60$ & 10 & 7,04 \\
\hline \multicolumn{3}{|l|}{ Categoria profissional } \\
\hline Técnicos de enfermagem & 60 & 42,25 \\
\hline Enfermeiros & 24 & 16,90 \\
\hline Médicos & 29 & 20,42 \\
\hline Médicos residentes & 17 & 12,97 \\
\hline Fisioterapeutas & 12 & 8,45 \\
\hline \multicolumn{3}{|l|}{ Setor de atuação } \\
\hline UTI adulto & 38 & 26,76 \\
\hline UTI neonatal & 50 & 35,21 \\
\hline Clínica médica (semi-intensivo) & 54 & 38,02 \\
\hline
\end{tabular}


Com relação à percepção de clima de segurança, não se observou diferença estatisticamente significante entre as categorias profissionais. Constatou-se que entre os trabalhadores da enfermagem e fisioterapeutas a percepção do clima de segurança foi menor (Tabela 2).

Os domínios percepção da gerência da unidade e do hospital e condições de trabalho foram representados pelos menores escores em todas as categorias profissionais observadas. Ressalta-se que a percepção do estresse obteve maior média entre os médicos residentes.

Quanto ao escore total obtido, nenhuma categoria apresentou valor positivo, exceto para o domínio satisfação no trabalho que obteve escore satisfatório somente entre os médicos residentes, conforme dados da Tabela 2 .

Tabela 2 - Distribuição dos domínios do Safety Attitudes Questionnaire (SAQ) por categorias profissionais. Região Centro-Oeste, Brasil, 2018 ( $n=142)$

\begin{tabular}{|c|c|c|c|c|c|c|}
\hline $\begin{array}{c}\text { Domínios } \\
\text { do SAQ }\end{array}$ & $\begin{array}{l}\text { Técnicos de } \\
\text { enfermagem }\end{array}$ & Enfermeiros & Médicos & $\begin{array}{l}\text { Médicos } \\
\text { residentes }\end{array}$ & Fisioterapeutas & $\begin{array}{c}p \\
\text { valor } \\
*\end{array}$ \\
\hline & $\begin{array}{l}\text { Média } \\
\text { (DP) }\end{array}$ & $\begin{array}{l}\text { Média } \\
\text { (DP) }\end{array}$ & $\begin{array}{l}\text { Média } \\
\text { (DP) }\end{array}$ & $\begin{array}{l}\text { Média } \\
\text { (DP) }\end{array}$ & $\begin{array}{l}\text { Média } \\
\text { (DP) }\end{array}$ & \\
\hline $\begin{array}{l}\text { Clima } \\
\text { trabalho } \\
\text { equipe }\end{array}$ & $\begin{array}{r}64,30 \\
(14,15)\end{array}$ & $\begin{array}{r}63,19 \\
(13,49)\end{array}$ & $\begin{array}{c}72,41 \\
(15,16)\end{array}$ & $\begin{array}{l}70,09 \\
(13,83)\end{array}$ & $\begin{array}{r}67,01 \\
(12,36)\end{array}$ & 0,07 \\
\hline $\begin{array}{l}\text { Clima } \\
\text { segu- } \\
\text { rança }\end{array}$ & $\begin{array}{l}54,64 \\
(15,15)\end{array}$ & $\begin{array}{l}52,08 \\
(12,09)\end{array}$ & $\begin{array}{c}57,38 \\
(15,15)\end{array}$ & $\begin{array}{l}59,24 \\
(12,37)\end{array}$ & $\begin{array}{c}50,89 \\
(16,05)\end{array}$ & 0,44 \\
\hline $\begin{array}{l}\text { Satisfação } \\
\text { trabalho }\end{array}$ & $\begin{array}{c}69,75 \\
(15,71)\end{array}$ & $\begin{array}{c}67,70 \\
(13,82)\end{array}$ & $\begin{array}{c}72,75 \\
(14,11)\end{array}$ & $\begin{array}{l}75,00 \\
(18,87)\end{array}$ & $\begin{array}{c}74,58 \\
(16,01)\end{array}$ & 0,42 \\
\hline $\begin{array}{l}\text { Percepção } \\
\text { estresse }\end{array}$ & $\begin{array}{c}68,85 \\
(16,37)\end{array}$ & $\begin{array}{l}71,09 \\
(17,94)\end{array}$ & $\begin{array}{c}71,76 \\
(18,94)\end{array}$ & $\begin{array}{c}80,51 \\
(18,47)\end{array}$ & $\begin{array}{c}60,41 \\
(19,27)\end{array}$ & 0,15 \\
\hline $\begin{array}{l}\text { Percepção } \\
\text { gerência }\end{array}$ & $\begin{array}{c}46,18 \\
(15,13)\end{array}$ & $\begin{array}{c}46,35 \\
(16,95)\end{array}$ & $\begin{array}{c}51,14 \\
(15,70)\end{array}$ & $\begin{array}{c}56,37 \\
(13,43)\end{array}$ & $\begin{array}{c}42,36 \\
(19,18)\end{array}$ & 0,14 \\
\hline
\end{tabular}




\begin{tabular}{lcccccc} 
Percepção & 45,97 & 46,18 & 47,55 & 50,98 & 45,13 & 0,82 \\
gerência & $(12,95)$ & $(13,28)$ & $(14,49)$ & $(16,23)$ & $(19,12)$ & \\
hospital & & & & & \\
Condições & 48,19 & 47,91 & 52,87 & 53,43 & 43,75 & 0,73 \\
trabalho & $(22,55)$ & $(20,74)$ & $(18,67)$ & $(20,63)$ & $(19,50)$ & \\
SAQ Total & 58,79 & 58,02 & 63,43 & 65,53 & 57,62 & 0,17 \\
& $(11,60)$ & $(10,37)$ & $(11,36)$ & $(9,54)$ & $(13,89)$ & \\
\hline
\end{tabular}

${ }^{*} p$ valor obtido pelo teste estatístico Kruskal-Wallis.

No que se refere aos gestores, majoritariamente eram do sexo feminino $(72,2 \%)$, chefes de unidades e setores $(85,2 \%)$, com vínculo institucional maior que 36 meses (74,1\%), tempo na ocupação atual inferir a 25 meses $(77,8 \%)$ e referiram experiência em campanhas de HM (72,2\%).

Quanto à percepção dos gestores a respeito dos impactos das IRAS na evolução clínica do paciente e nas despesas do hospital, um total de $91,7 \%$ relatou que existe alto impacto em ambas, embora 5,5\% dos gestores julgaram que a prática de HM na prevenção de IRAS possui baixa eficácia e 9,3\% perceberam essa medida como de baixa importância nas prioridades da gerência institucional (Tabela 3).

Tabela 3 - Distribuição da frequência (f) dos gestores em relação ao impacto das IRAS, eficácia e importância da Higiene das Mãos na prevenção das Infecções Relacionadas à Assistência à Saúde.

Região Centro-Oeste, Brasil, $2018(n=54)$

\begin{tabular}{lll}
\hline Variáveis & $f$ & $\%$ \\
\hline
\end{tabular}

Impacto das IRAS* na evolução clínica do paciente

Muito baixo

Baixo

Alto

Muito alto

Impacto das IRAS nas despesas do hospital

Muito baixo

0

0

Baixo

6 


\section{Eficácia da HM † na prevenção das IRAS}

$\begin{array}{lcc}\text { Muito baixo } & 1 & 1,9 \\ \text { Baixo } & 2 & 3,7 \\ \text { Alto } & 14 & 25,9 \\ \text { Muito alto } & 37 & 68,5\end{array}$

Importância da $\mathrm{HM} †$ nas prioridades da gerência da sua instituição

Muito baixo

0

0

Baixo

5

Alto

* IRAS - Infecções Relacionadas à Assistência à Saúde † HM - Higiene das Mãos

Ao serem questionados sobre a taxa de IRAS, 13\% afirmaram não haver índice das referidas infecções na instituição e 50\% dos gestores relataram um índice superior a $15 \%$.

No tocante à percepção do percentual de profissionais de saúde que realizam a HM, 33\% dos gestores julgaram que apenas $50 \%$ do total de profissionais realizam essa prática conforme os momentos recomendados. Além disso, um total de 50\% avaliou que os trabalhadores de saúde não se esforçam o suficiente para realizar a HM, tampouco recebem de forma positiva o pedido para realizá-la (54\%).

Com relação à eficácia de estratégias educativas para aumento permanente da adesão às práticas de $\mathrm{HM}$, evidenciou-se que algumas ações foram menos percebidas como eficazes pelos gestores em detrimento de outras, como o feedback do desempenho do profissional referente à adesão à HM e a participação do paciente como estratégia para a adesão a essa prática (Tabela 4). 
Tabela 4 - Distribuição da frequência (f) dos gestores em relação à eficácia das ações para realização da HM. Região Centro-Oeste, Brasil, 2018 ( $n=54)$

$\begin{array}{lll}\text { Variáveis } & f & \%\end{array}$

Líderes e gerentes executivos que apoiam e promovem abertamente a $\mathrm{HM}^{*}$

Ineficaz

0

0

Pouco eficaz

4

7,4

Nem eficaz, nem ineficaz

8

14,8

Eficaz

40,7

Muito eficaz

20

37,0

Disponibilização de preparações alcoólicas para a HM

Ineficaz

0

0

Pouco eficaz

5

9,3

Nem eficaz, nem ineficaz

4

7,4

Eficaz

20

37,0

Muito eficaz

25

46,3

Cartazes sobre como realizar a HM expostos no ponto de assistência como lembretes

Ineficaz

Pouco eficaz

Nem eficaz, nem ineficaz

Eficaz

Muito eficaz

Ineficaz

Pouco eficaz

Nem eficaz, nem ineficaz
1

1,8

3

13

24,0

24

44,4

13

24,0

1

1,8

3

5,5

13

24,0 
Profissional de saúde treinado em como realizar a HM

Ineficaz

Pouco eficaz

Nem eficaz, nem ineficaz

Eficaz

Muito eficaz

Instruções claras e simples visíveis sobre a HM

Ineficaz

Pouco eficaz

Nem eficaz, nem ineficaz

Eficaz

Muito eficaz
1

2

11

23

17
1,8

20,4

46,6

31,5

\section{Feedback aos profissionais de saúde sobre o desempenho em HM}

Ineficaz

Pouco eficaz

Nem eficaz, nem ineficaz

Eficaz

Muito eficaz
10

11

20,4

13

24,07

11

20,4

9
40,7

27,8

Pacientes são estimulados a lembrar os profissionais de saúde a realizar a HM

Ineficaz

Pouco eficaz

Nem eficaz, nem ineficaz

Eficaz

Muito eficaz
13

11

12

12

6
24,0

20,4

22,2

22,2

11,1 


\section{DISCUSSÃO}

A amostra deste estudo foi composta majoritariamente por participantes do sexo feminino ( $\mathrm{n}=97 ; 68,30 \%$ ), com média de 31 a 40 anos de idade. A maioria dos trabalhadores compunha a equipe de enfermagem $(n=84 ; 59,15 \%)$ e o setor com maior número de participantes foi a clínica médica (semi-intensivo) $(\mathrm{n}=54 ; 38,02 \%)$.

Os resultados da presente investigação mostraram a fragilidade nos aspectos relacionados ao clima de segurança do paciente, uma vez que os escores dos domínios foram negativos, isto é, menores que 75 pontos, cujo achado está em consonância com estudos realizados em outros estados brasileiros ${ }^{6-8}$

Merece destaque a avaliação da percepção da gerência da unidade e do hospital, que reflete a percepção dos profissionais da assistência sobre o reconhecimento e a aprovação das ações da gerência para a segurança do paciente na instituição. Estes foram os domínios que apresentaram menores escores quando comparados aos demais, corroborando com estudos nacionais ${ }^{6-8}$ internacionais $9-10$

Os resultados do presente estudo mostraram o distanciamento da gerência quanto às questões relacionadas à segurança do paciente, percebido pelos profissionais assistenciais das unidades críticas, locais esses em que os índices de IRAS são alarmantes mundialmente ${ }^{11}$. Por outro lado, os gestores perceberam baixo comprometimento dos trabalhadores de saúde com a HM. Em outras palavras, essa dualidade pode repercutir em práticas inseguras, em especial no que diz respeito à prevenção de IRAS e, portanto, práticas de assistência em saúde seguras.

Percepções baixas e limitadas do clima de segurança e da importância da prática de HM com vistas à prevenção das IRAS são incongruentes com uma assistência segura e de qualidade. Tais resultados sugerem que há uma necessidade de fortalecer os papéis de liderança dos gerentes visando à integração com a equipe de saúde ${ }^{12-13}$. Isso é crucial, uma vez que o trabalho em saúde é sabidamente desenvolvido por múltiplos agentes, e a valorização da média e alta gerência a respeito de ações de segurança é, possivelmente, um elemento crítico para a concretude do cuidado seguro $^{13}$

No que se refere ao domínio condições de trabalho, os baixos escores evidenciados no presente estudo podem estar influenciados também por fatores como longas jornadas de trabalho, inadequado dimensionamento de pessoal, deficit na infraestrutura do local, dentre outros fatores 
que contribuem para o desgaste profissional e favorecem a ocorrência de erros durante a assistência $^{14-15}$ Nesse contexto, futuros estudos devem ser conduzidos diante desse diagnóstico encontrado.

Quanto ao domínio percepção do estresse, os resultados mostraram que houve reconhecimento dos fatores estressores que podem influenciar na execução do trabalho da equipe de enfermagem, médicos e médicos residentes.

Destaca-se que os profissionais de enfermagem geralmente possuem duplo vínculo empregatício e ainda conciliam sua jornada com os afazeres domésticos, bem como buscam por melhor qualificação profissional, o que resulta em um cotidiano de atividades intensas. Desse modo, o descanso fica comprometido, o que pode refletir no desenvolvimento do estresse, depressão e Síndrome de Burnout nesses trabalhadores ${ }^{15}$.

Com relação à percepção de estresse evidenciada por médicos residentes, estudos mostram que residência é um dos períodos mais críticos da educação para a qualificação médica devido à necessidade de estudo persistente, responsabilidade excessiva, altas demandas de atendimento, horário de trabalho prolongado e falta de tempo para descansar, que são elementos que levam ao esgotamento, depressão e estresse ocupacional ${ }^{16-17}$.

Outra lacuna importante evidenciada na presente investigação refere-se ao desconhecimento por parte de alguns gestores dos elementos-chave da estratégia multimodal, tais como o feedback da prática, cartazes no local de trabalho e envolvimento do paciente. Estudos têm demonstrado que quando os gestores apoiam a referida estratégia e se sentem corresponsáveis pela implementação de todos seus componentes integradores, obtêm-se maiores índices de adesão à HM e só assim os mesmos podem ser sustentados ao longo do tempo ${ }^{4}$

No presente estudo, embora os gestores tenham percebido a eficácia da higienização das mãos no controle das IRAS, menos de $70 \%$ dos gestores reconheceram os lembretes nos locais de trabalho e o feedback do desempenho de HM como ações eficazes e impactantes no estímulo à adesão dessa prática na instituição investigada . Todavia, a OMS reconhece e divulga essas estratégias como componentes-chave da estratégia multimodal para alcance de resultados positivos em saúde ${ }^{5}$.

Estudo recente de revisão integrativa evidenciou que intervenções utilizando todos os elementos da estratégia multimodal de forma articulada e interdependente aumentaram a adesão à $\mathrm{HM}$, destacando que alguns componentes foram fundamentais para manter a sustentabilidade ao 
longo do tempo, tais como a participação e o envolvimento da gerência com medidas de segurança e o feedback aos trabalhadores de saúde sobre seu desempenho em $\mathrm{HM}^{4}$.

No que concerne à inclusão do paciente na implementação de práticas seguras, o Programa Nacional de Segurança do Paciente, criado no ano de $2013^{1}$, ressalta a importância da participação do paciente, familiares e acompanhantes no processo de cuidado e na adoção de estratégias para adesão à HM. Tal eixo refere-se ao envolvimento do cidadão em sua segurança para prevenção de erros e EA em saúde, assim como no alcance das metas internacionais de segurança do paciente, como a redução das IRAS nos serviços de saúde ${ }^{2,18}$.

Neste estudo, faz-se importante ressaltar que, para os gestores $(66,6 \%)$, a ajuda do paciente para lembrar os trabalhadores de saúde da HM não foi considerada eficaz. No entanto, pesquisa realizada em Genebra destacou que a participação e o envolvimento do paciente na implantação da estratégia multimodal para HM foram considerados componentes existosos, uma vez que os índices de adesão apresentaram melhora substancial ${ }^{18}$.

Ainda nesse contexto, pesquisas que investigaram a percepção do profissional de saúde sobre a importância do envolvimento do paciente para maior adesão às práticas de HM mostraram concordância nesse envolvimento, com índices de aprovação representados por cerca de 93,3\% e $62,8 \%$, respectivamente ${ }^{19-20}$. Por outro lado, os principais motivos alegados pelos trabalhadores de saúde em não envolver o paciente referiram-se ao dever do profissional de saúde com essa prática e os efeitos negativos em seu vínculo com o paciente ${ }^{20}$.

Destaca-se como limitação do presente estudo a realização da pesquisa em um único hospital, em setores críticos, com população e consequente amostra reduzida para algumas categorias profissionais, o que torna comprometida a generalização dos resultados encontrados. Ademais, outros fatores devem ser investigados como contribuintes para a percepção reduzida de clima de segurança institucional do paciente e futuras pesquisas devem ser realizadas a fim de analisar possíveis fatores associados.

\section{CONCLUSÃO}

Conclui-se que os trabalhadores de saúde apresentaram baixa percepção de clima de segurança na instituição investigada, com escores inferiores ao preconizado em todos os domínios avaliados, assim como os gestores tiveram uma percepção limitada sobre higiene das mãos e infecções relacionadas à assistência à saúde. Essa é uma questão que pode repercutir em práticas inseguras, reforçada pelo fato de que a percepção da gerência foi o domínio do clima de segurança com menores valores em todas as categorias profissionais avaliadas, o que reflete com clareza o 
distanciamento entre equipe assistencial e gerencial quanto às questões relacionadas à segurança do paciente.

Faz-se necessário evidenciar que investimentos em HM sem o comprometimento da gestão devem ser questionados quando se pretende lograr melhores índices de adesão, pois a cultura de segurança eficaz é componente transversal a toda estratégia multimodal. Da mesma forma, não se pode haver envolvimento substancial e concreto da gestão sem a conscientização desses líderes sobre a interdependência e complementaridade de componentes importantes que permeiam a estratégia multimodal, pouco conhecidos por eles, tais como feedback das práticas de HM, participação do paciente, dentre outros.

O estudo contribui para prática no sentido de reforçar o clima de segurança do paciente como escopo passível de mensuração pela equipe de saúde, em especial os trabalhadores da equipe de enfermagem, o que sem dúvida é relevante para o incremento de estratégias factíveis para o cuidado seguro. Além disso, a dualidade na percepção de clima de segurança do paciente entre trabalhadores com a percepção da efetividade da higiene das mãos entre gestores é uma contribuição que evidencia que a segurança do paciente é um bem completo e multidimensional na dinâmica da organização.

\section{CONTRIBUIÇÕES}

Informa-se que todos os autores contribuíram igualmente na concepção do projeto de pesquisa, coleta, análise e discussão dos dados, bem como na redação e revisão crítica do conteúdo com contribuição intelectual e na aprovação da versão final do estudo.

\section{CONFLITO DE INTERESSES}

Nada a declarar.

\section{REFERÊNCIAS}

1. Ministério da Saúde (Brasil). Gabinete do Ministro. Portaria MS/GM n 529, de 1 de abril de 2013. Disponível em:

http://bvsms.saude.gov.br/bvs/saudelegis/gm/2013/prt0529_01_04_2013.html.

2. The Joint Commission. 2018 Hospital National Patient Safety Goals. Illinois: The Joint Commission, 2019 [acesso em 13 abr 2019]; Disponível em:

https://www.jointcommission.org/assets/1/6/2018_HAP_NPSG_goals_final.pdf

3. Centers for Disease Control and Prevention. National and state healthcare associated Infections (HAl) progress report. Atlanta (US): CDC, 2016.

4. Valim MD, Rocha ILS, Souza TPM, Cruz YA, Bezerra TB, Baggio E, et al. Eficácia da estratégia multimodal para adesão à higiene das mãos: revisão integrativa. Rev Bras Enferm. [Internet]. 2019;72(2). DOI: https://doi.org/10.1590/0034-7167-2018-0584

5. World Health Organization (WHO). A Guide to the Implementation of the WHO Multimodal 
Hand Hygiene Improvement Strategy. Geneva: WHO; 2009. 48p.

6. Luiz RB, Simões ALA, Barichello E, Barbosa MH. Factors associated with the patient safety climate at a teaching hospital. Rev. Latino-Am. Enfermagem [Internet]. 2015;23(5):880-887. DOI: $10.1590 / 0104-1169.0059 .2627$

7. Barbosa MH, Floriano DR, Oliveira KF, Nascimento KG, Ferreira LA. Clima de segurança do paciente de um hospital privado. Texto Contexto Enferm. [Internet] 2016; 25(3):e1460015. DOI: https: / / doi.org/10.1590/0104-07072016001460015

8. Carvalho REFL, Cassiani SHB. Cross-cultural adaptation of the Safety Attitudes Questionnaire - Short Form 2006 for Brazil. Rev. Latino-Am. Enfermagem. [Internet].2012; 20(3):575-82. DOI: https:// doi.org/10.1590/S0104-11692012000300020

9. Dunstan E, Coyer F. Safety culture in two metropolitan Australian tertiary hospital intensive care units: A cross-sectional survey. Australian Critical Care [Internet].2019; 33(1): 4-11 DOI:10.1016/j.aucc.2018.11.069

10. Hayashi R, Fujita S, lida, S, Nagai Y, Shimamor, Y, \& Hasegawa T. Relationship of patient safety culture with factors influencing working environment such as working hours, the number of night shifts, and the number of days off among healthcare workers in Japan: a crosssectional study. BMC health services research [Internet] 2020; 20(1), 310. DOI: 10.1186/s12913-020-05114-8

11. Baviskar AS, Khatib KI, Rajpal D, Dongare HC. Nosocomial infections in surgical intensive care unit: A retrospective single-center study. Int J Crit Illn Inj Sci. [Internet] 2019; 9(1):16-20. DOI: 10.4103/IJCIIS.IJCIIS_57_18

12. Silva VLS, Camelo SHH, Soares MI, Resck ZMR, Chaves LDP, Santos FC, et al. Práticas de liderança em enfermagem hospitalar: uma self de enfermeiros gestores. Rev Esc Enferm USP. [Internet] 2016; 207(51):e03206. DOI: http://dx.doi.org/10.1590/S1980-220X2016024403206

13. Kawamoto AM, Oliveira JLC, Tonini NS, Nicola AL. Liderança e cultura de segurança do paciente: percepções de profissionais em um hospital universitário. J. res. fundam. care. [Internet] 2016 [acesso em 04 abr 2019]; 8(2):4387-4398. Disponível em: http://www.seer.unirio.br/index.php/cuidadofundamental/article/view/4530

14. Atefi N, Abdullah KL, Wong LP, Mazlom R. Factors influencing registered nurses perception of their overall job satisfaction: a qualitative study. Int Nurs Rev. [Internet] 2014; 61(3):352-60. DOI: http: / /dx.doi.org/10.9789/2175-5361.2016.v8i2.4387-4398

15. Rodrigues CCFM, Santos VEP, Sousa P. Segurança do paciente e enfermagem: interface com estresse e Síndrome de Burnout. Rev. Bras. Enferm. 2017; 70(5): 1083- 1088. DOI: https: / / doi.org/10.1590/0034-7167-2016-0194

16. Rosen T, Zivin K, Eisenberg D, Guille C, Sen S. The Cost of Depression-Related Presenteeism in Resident Physicians. Acad Psychiatry . 2018; 42 (1): 84-87. DOI: 10.1007 / s40596-0170867-1

17. Trockel M, Bohman B, Lesure E, et al. A Brief Instrument to Assess Both Burnout and Professional Fulfillment in Physicians: Reliability and Validity, Including Correlation with SelfReported Medical Errors, in a Sample of Resident and Practicing Physicians. Acad Psychiatry. 2018;42(1):11-24. doi:10.1007/s40596-017-0849-3

18. Oliveira AC, Pinto AS. Participação do paciente na higienização das mãos entre profissionais de saúde. Rev Bras Enferm. 2018;71(2):259-64. DOI:10.1590/0034-7167-2016-0124

19. Hand Hygiene: A Handbook for Medical Professionals, First Edition. 2017; John Wiley \& Sons, p. 18-27. DOI: https://doi.org/10.1002/9781118846810.ch4

20. Kim MK, Nam EY, Na SH, Shin MJ, Lee HS, Kim NH, et al. Discrepancy in perceptions regarding patient participation in hand hygiene between patients and health care workers. Am J Infect Control [Internet]. 2015; 43(5):510-5. DOI: 10.1016/j.ajic.2015.01.018 


\section{Correspondência}

THAISSA BLANCO BEZERRA

E-mail: thaissa_blanco@hotmail.com

Submissão: $16 / 08 / 2020$

Aceito: 19/04/2021

Copyright $\odot 2021$ Revista de Enfermagem UFPE on line/REUOL.

(cc) EY Este é um artigo de acesso aberto distribuído sob a Atribuição CC BY 4.0 Creative Commons AttributionShareAlike 4.0 International License, a qual permite que outros distribuam, remixem, adaptem e criem a partir do seu trabalho, mesmo para fins comerciais, desde que lhe atribuam o devido crédito pela criação original. É recomendada para maximizar a disseminação e uso dos materiais licenciados. 